\title{
Primes are No Longer Lonely: A Tale of Twin Primes and the Devoted Chinese Mathematician Yitang Zhang *
}

\author{
by Lizhen Ji \\ Department of Mathematics, University of Michigan
}

What is mathematics? Kronecker said: "God made the integers, all the rest is the work of man." What makes integers? Prime numbers! Indeed, every integer can be written, essentially uniquely, as a product of primes. Since ancient Egypt (around 3000 BC), people have been fascinated with primes. Today, large prime numbers have become important in modern cryptography.

More than two thousand years ago, Euclid proved that there are infinitely many primes, but people observed that primes occur less and less frequently. The celebrated twin prime conjecture says the extremal exceptions can occur, i.e., there are infinitely many pairs of primes with gaps equal to 2. The first real breakthrough to this century old problem was made by the Chinese mathematician Yitang Zhang: There are infinitely many pairs of primes each of which is separated by at most 70 millions.

\section{Prime numbers}

The story of primes is long and complicated, and the story of Zhang is touching and inspiring. In some sense, the history of primes gives an accurate snapshot of the history of mathematics, and many major mathematicians have been attracted to them.

After Euclid, the written history of prime numbers lay dormant until the 17th century when Fermat stated that all numbers of the form $2^{2^{n}}+1$ (for natural numbers $n$ ) are prime. He did not prove it but he checked it for $n=1, \ldots, 4$. Fermat's work motivated Euler and many others.

\footnotetext{
" I would like to thank Prof. Shing-Tung Yau for suggesting the title of this article, Prof. William Dunham for information on the history of the twin prime conjecture, Prof. Liming Ge for biographic information about Yitang Zhang, Prof. Shiu-Yuen Cheng for pointing out the paper of Soundararajan cited in this article, Prof. Lo Yang for information about Chengbiao Pan quoted below, Prof. Yuan Wang for detailed information on results related to the twin prime conjecture, and Prof. John Coates for reading this article carefully and for making several valuable suggestions and sharing the pictures of a letter by Gauss on the prime number theorem.
}

For example, Euler showed that the next Fermat number $2^{2^{5}}+1$ is not prime. This shows the danger of asserting a general statement after few experiments. Euler worked on many different aspects of prime numbers. For example, his correspondences with Goldbach in 1742 probably established the Goldbach conjecture as a major problem in number theory. In his reply, Euler wrote "every even integer is a sum of two primes. I regard this as a completely certain theorem, although I cannot prove it."

Distribution of primes is a natural and important problem too and has been considered by many people. Near the end of the 18th century, after extensive computations, Legendre and Gauss independently conjectured the Prime Number Theorem: As x goes to infinity, the number $\pi(x)$ of primes up to $x$ is asymptotic to $x / \ln (x)$.

Gauss never published his conjecture (although a long letter dated 1849, explaining precisely what he had discovered exists in the Göttingen Library), though Legendre did, and a younger colleague of Gauss, Dirichlet, came up with another formulation of the theorem. In 1850, Chebyshev proved a weak version of the prime number theorem which says that the growth order of the counting function $\pi(x)$ is as predicted, and derived as a corollary that there exists a prime number between $n$ and $2 n$ for any integer $n \geq 2$. (Note that the length of the period goes to infinity).

Though the zeta function was used earlier in connection with primes in papers of Euler and Chebyshev, it was Riemann who introduced the zeta function as a complex function and established close connections between the distribution of prime numbers and the location of complex zeros of the Riemann zeta function. This blending of number theory and complex analysis has changed number theory forever, and the Riemann hypothesis on the complex zeros of the Riemann zeta function is still open and probably the most famous problem in mathematics.

In his 1859 paper, Riemann sketched a program to prove the prime number theorem via the Riemann zeta 
function, and this outline was completed independently by Hadamard and de la Vallée Poussin in 1896.

One unusual (or rather intriguing, interesting) thing about primes is that they exhibit both regular and irregular (or random) behavior. For example, the prime number theorem shows that its overall growth follows a simple function, but gaps between them are complicated and behave randomly (or chaotically). One immediate corollary of the prime number theorem is that gaps between primes go to infinity on average (or the density of primes among integers is equal to zero). Understanding behaviors of these gaps is a natural and interesting problem. (Note that in some ways, this also reflects the difficulty in handling the error terms in the asymptotics of the counting function $\pi(x)$ of primes, which are related to the complex zeros of the Riemann zeta function as pointed out by Riemann).

\section{Gaps between primes}

What is the written history of the study on gaps between primes? In 1849, Alphonse de Polignac (1817-1890) conjectured that every even integer can occur as gaps of infinitely many pairs of consecutive primes, and the case of 2, i.e., the existence of infinitely many pairs of twin prime pairs, is a special case. The twin prime number in the current form was stated by James W. L. Glaisher (1848-1928) in 1878, who was Second Wrangler in 1871 in Cambridge, a number theorist, the tutor of a famous philosopher Ludwig Wittgenstein, and President of the Royal Astronomical Society. After counting pairs of twin primes among the first few millions of natural numbers, Glaisher concluded: "There can be little or no doubt that the number of prime-pairs is unlimited; but it would be interesting, though probably not easy, to prove this."

These were the first known instances where the twin prime conjecture was stated. Given the simple form and naturalness of the twin prime conjecture, it might be tempting to guess that this question might be considered by people earlier. It might not be a complete surprise if it were considered by the Greeks already. But according to experts on the history of mathematics, in particular on the work of Euler, there was no discussion of twin primes in the work of Euler. Since Euler was broad and well versed in all aspects of number theory, one might conjecture that Polignac was the first person who raised the question on twin primes.

This easily stated conjecture on twin primes has been attacked by many people. Though the desired gap 2 is the dream, any estimates on the gaps between primes smaller than the obvious one from the prime number theorem is valuable and interesting, and any description or structure of distribution of these gaps is important and interesting as well. ${ }^{1}$

\footnotetext{
1 The problem on spacing between prime numbers is one of several problems concerning spacing in naturally occurring sequences such as zeros of the Riemann zeta-function, energy
}

Many partial and conditional results have been obtained on sizes of gaps between primes, and there are also a lot of numerical work listing twin prime pairs. Contributors to this class of problems include Hardy, Littlewood, Siegel, Selberg, Rankin, Vinogradov, Hua, Erdös, Bombieri, Brun, Davenport, Rademacher, Rényi, Yuan Wang, Jingrun Chen, Chengdong Pan, Friedlander, Iwaniec, Heath-Brown, Huxley, Maier, Granville, Soundararajan. Indeed, it might be hard to name many great analytic number theorists in the last 100 years who have not tried to work on the twin prime conjecture directly or indirectly. Of course, there are many attempts by amateur mathematicians as well.

One significant and encouraging result was proved in 2009 by Goldston, Pintz, and Yildirim. In some sense, they started the thaw of the deep freeze. They showed that though gaps between primes go to infinity on average, they can be exceptionally small. This is the result of "culminating 80 years of work on this problem" [2, p. 1].

Under a certain condition called the Elliott-Halberstam conjecture on distribution of primes in arithmetic progressions, they can prove that there are infinitely many pairs of primes with gaps less than 16 . Though this condition might be "within a hair's breadth" of what is known [1, 822], it seems to be hard to check.

In [1, p. 822], they raised "Question 1. Can it be proved unconditionally by the current method that there are, infinitely often, bounded gaps between primes?"

How to make use of or improve results in [1]? Probably this was the starting point for Zhang. But problems of this kind are hard [1, p. 819]: "Not only is this problem believed to be difficult, but it has also earned the reputation among most mathematicians in the field as hopeless in the sense that there is no known unconditional approach for tackling the problem."

Indeed, difficulties involved seemed insurmountable to experts in the field before the breakthrough by Zhang. According to Soundararajan [3, p. 17]:

"First and most importantly, is it possible to prove unconditionally the existence of bounded gaps between primes? As it stands, the answer appears to be no, but perhaps suitable variants of the method will succeed."

The most basic, or the only method, to study prime numbers is the sieve method. But there are many different sieves with subtle differences between them, and it is an art to design the right sieve to attack each problem. Real original ideas were needed to overcome the seeming impasse. After working on the problem for three years, one key revelation occurred to Zhang when he was visiting a friend's house in July 2012, and he solved Question 1 in [1]. In some sense, his persistence and confidence allowed him to succeed where all world experts failed and gave up. He also handled this extreme difficulty with the ease and

levels of large nuclei, the fractional parts of $\sqrt{n}$ for $n \in N$. One question asks whether the spacings can be modelled by the gaps between random numbers (or eigenvalues of randomly chosen matrices), or whether they follow other more esoteric laws. 
steadiness of an old hand. Henryk Iwaniec, one of the world leading experts on analytic number theory, has written:

Yitang Zhang was not well-known to specialists in number theory before his fantastic paper on prime numbers was recognized by the Annals of Mathematics three weeks ago. But he possessed the knowledge of the most sophisticated areas of analytic number theory, and he could use it all with ease. Also, he was able to make a breakthrough where many investigators were stuck, not because something little was overlooked, but because of new, clever arrangements which he introduced and brilliantly executed. You could sense immediately that the work had a great chance to be fine by looking at the clear and logical architecture of the arguments. It does not mean the paper is simple or elementary. To the contrary, the work of Zhang constitutes the state-of-the art of analytic number theory. It also borrows gracefully from other areas, for example, it makes use indirectly of the Riemann hypothesis for varieties over finite field. Zhang's work will trigger a lasting avalanche of refinements and improvements with many innovations. Overnight Zhang redirected the focus of analytic number theory. How long do we need to wait to see what comes next?

On May 13, 2013, Zhang gave a seminar talk at Harvard University upon the invitation of Prof. Shing-Tung Yau. At the seminar, he announced to the world his spectacular result in [5]: There exist infinitely many pairs of primes with gaps less than 70 millions.

This marks the end of a long triumphant period in analytic number theory and could be the beginning of a new period for the subject.

\section{Zhang's career}

Zhang's academic career is a mingling of the standard and the nonstandard, maybe similar to prime numbers he loves. He went to Peking University (PKU) in 1978 and graduated from college as the top student in $1982 .{ }^{2}$ Then in 1982-1985, he continued to study for the Master degree at Peking University under Chengbiao Pan and hence was an academic grandson of Loo-Keng Hua (Luogeng Hua, 1910-1985). ${ }^{3}$ After receiving a Ph.D. degree at Purdue

\footnotetext{
${ }^{2}$ This seems to be the unanimous opinion of former students from PKU who knew him.

${ }^{3}$ Though Chengbiao Pan was not a student of Hua formally, the influence of Hua on Pan was huge and clearly visible. According to Prof. Lo Yang: "Chengbiao Pan was an undergraduate student in PKU (1955-1960). He worked, after 1960, in Beijing College on Mechanization of Agriculture, and became a professor of Beijing Agriculture University in the eighties, after his college was combined in this university. Though Pan was the professor of Beijing Agriculture University, he spent most time in PKU. Pan was a student of Prof. Szu-Hao Min (Sihe Min, 1913-1973), but he did not study number theory at that time. Instead, Pan studied the generalized analytic functions (in the sense of L. Bers and the
}

University in $1991,{ }^{4}$ he could not get a regular academic job and worked in many areas at many places including accounting firms and fast food restaurants. But mathematics has always been his love. From 1999 to 2005, he acted as a substitute or taught few courses at University of New Hampshire. From 2005 to present, he has been a lecturer there and is an excellent teacher, highly rated by students. In some sense, he has never held as a regular research position in mathematics up to now. It is impressive and touching that he has been continuing to do research on the most challenging problems in mathematics (such as the zeros of the Riemann zeta function and the twin prime conjectures) in spite of the difficult situation over a long period. His persistence has paid off as in the Chinese saying: huang tian bu fu you xin ren (Heaven never disappoints those determined people, or Heaven helps those who help themselves!)

His thesis dealt with the famous Jacobian conjecture on polynomial maps, which is also infamous for many false proofs and is still open. After obtaining his Ph.D. degree and before this breakthrough on twin primes, Zhang published only one paper, "On the zeros of $\zeta^{\prime}(s)$ near the critical line" [4] in the prestigious Duke Journal of Mathematics, which studies zeroes of the Riemann zeta function and its derivative and gaps between the zeros. In 1985, Zhang published another paper on zeros of the Riemann zeta function in Acta Mathematica Sinica, one of the leading mathematics journals in China.

It is perhaps helpful to point out that spacing of these zeros and twin primes are closely related [3, p. 2]: "Precise knowledge of the frequency with which prime pairs $p$ and $p+2 k$ occur (for an even number $2 k$ ) has subtle implications for the distribution of spacings between ordinates of zeros of the Riemann zeta-function... Going in the other direction, weird (and unlikely) patterns in zeros of zeta-like functions would imply the existence of infinitely many twin primes."

In the current culture of mass production of everything, probably one sobering question is how much one should or can really produce. (This reminds one of a famous short story by Tolstoy, "How Much Land Does A Man Need?" One can also replace "land" by other attractive items, and "much" by "many.")

Is counting papers and number of pages an effective criterion? Probably one should also keep in mind that the

Russian mathematician Vekywa), since Prof. Min was forced to change his field from number theory to this area in 1958. I believe that Pan's knowledge and ability in number theory was mainly due to his elder brother Chengdong Pan. Chengdong Pan was the graduate student of Prof. Min (1956-1959). But he was also considered to be a student of Prof. Hua, especially in the research of Goldbach conjecture. In fact, Prof. Min was also much influenced by Prof. Hua."

${ }^{4}$ Around 1984, Prof. Shing-Tung Yau tried to arrange for Zhang to go to UC San Diego to study with the well-known analytic number theorist Harold Stark there. Unfortunately, for some unknown reason, this idea was apparently vetoed in China. Otherwise he might have moved academically along a path which is closer to a geodesic. 
best judgement on everything under the Sun is still the time!

Maybe not everyone is familiar with the Riemann hypothesis, but every student who has studied calculus will surely have heard of Riemann and his integrals. Many mathematicians will agree that Riemann is one of the greatest mathematicians in the history. But it is probably

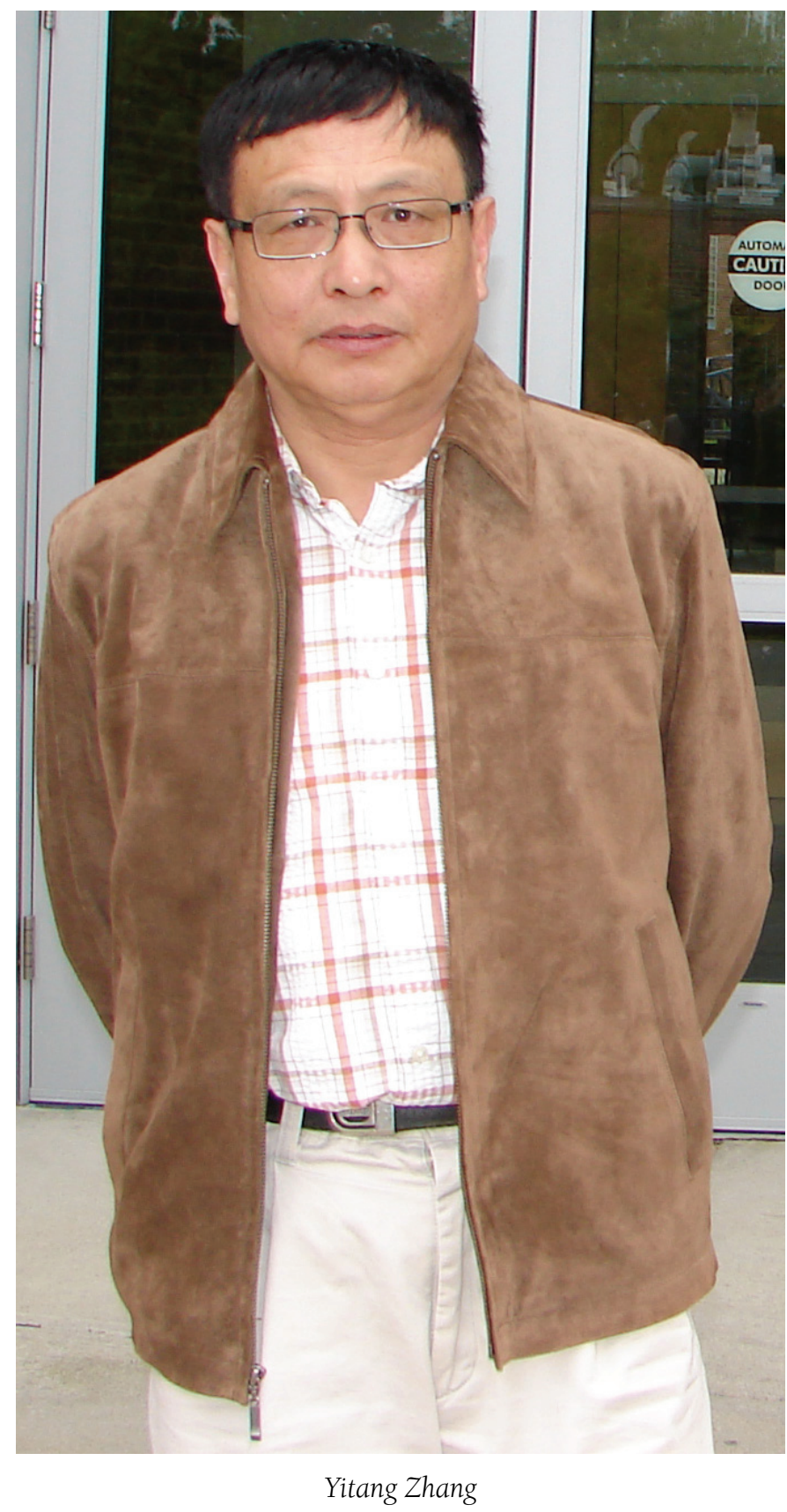

less known that in his life time, Riemann only published five papers in mathematics together with four more papers in physics. (Galois published fewer papers in his life time, but he never worked as a full time mathematician and died at a very young, student age.)

\section{Primes are not loners}

The concept of primes is also a sentimental one. Primes are lonely numbers among integers, but for some primes (maybe also for some human beings), one close partner as in twin prime pairs is probably enough and the best. This sentiment is well described in the popular novel, "The Solitude of Prime Numbers" by Paolo Giordano. Primes, he writes in this book, are

...suspicious, solitary numbers, which is why Mattia [the hero of the novel] thought they were wonderful. Sometimes he thought that they had ended up in that sequence by mistake, that they'd been trapped, like pearls strung on a necklace. Other times he thought that they too would have preferred to be like all the others, just ordinary numbers, but for some reasons they couldn't do it... among prime numbers, there are some that are even more special. Mathematicians call them twin primes: pairs of prime numbers that are close to each other, almost neighbors, but between them there is always an even number that prevents them from touching.

Zhang reminds people one of the heroes of several generations of Chinese students, Jingrun Chen, and his work on the famous Goldbach conjecture. Chen and Zhang both worked persistently and lonely on deep problems in number theory, and they both brought glory to China, in particular, to the Chinese mathematics community.

Of course, the story of Jingrun Chen is well-known to almost every Chinese student. For a romantic rendition of a mathematician's pursuit of the Goldbach conjecture, one might enjoy reading the book "Uncle Petros and Goldbach's Conjecture: A Novel of Mathematical Obsession" by Apostolos Doxiadis. (Incidentally, the hero in this novel, Uncle Petros, published only one paper after his Ph.D. degree and before he switched to work on the Goldbach conjecture.)

\section{References}

[1] D. Goldston, J. Pintz, C. Yldrm, Primes in tuples. I. Ann. of Math. (2) 170 (2009), no. 2, 819-862.

[2] D. Goldston, J. Pintz, C. Yldrm, Primes in tuples. II. Acta Math. 204 (2010), no. 1, 1-47.

[3] K. Soundararajan, Small gaps between prime numbers: the work of Goldston-Pintz-Yldrm. Bull. Amer. Math. Soc. (N.S.) 44 (2007), no. 1, 1-18.

[4] Y. Zhang, On the zeros of $\zeta^{\prime}(s)$ near the critical line. Duke Math. J. 110 (2001), no. 3, 555-572.

[5] Y. Zhang, Bounded gaps between primes, preprint, 2013, 56 pages. 


\section{Appendix: Photographs}
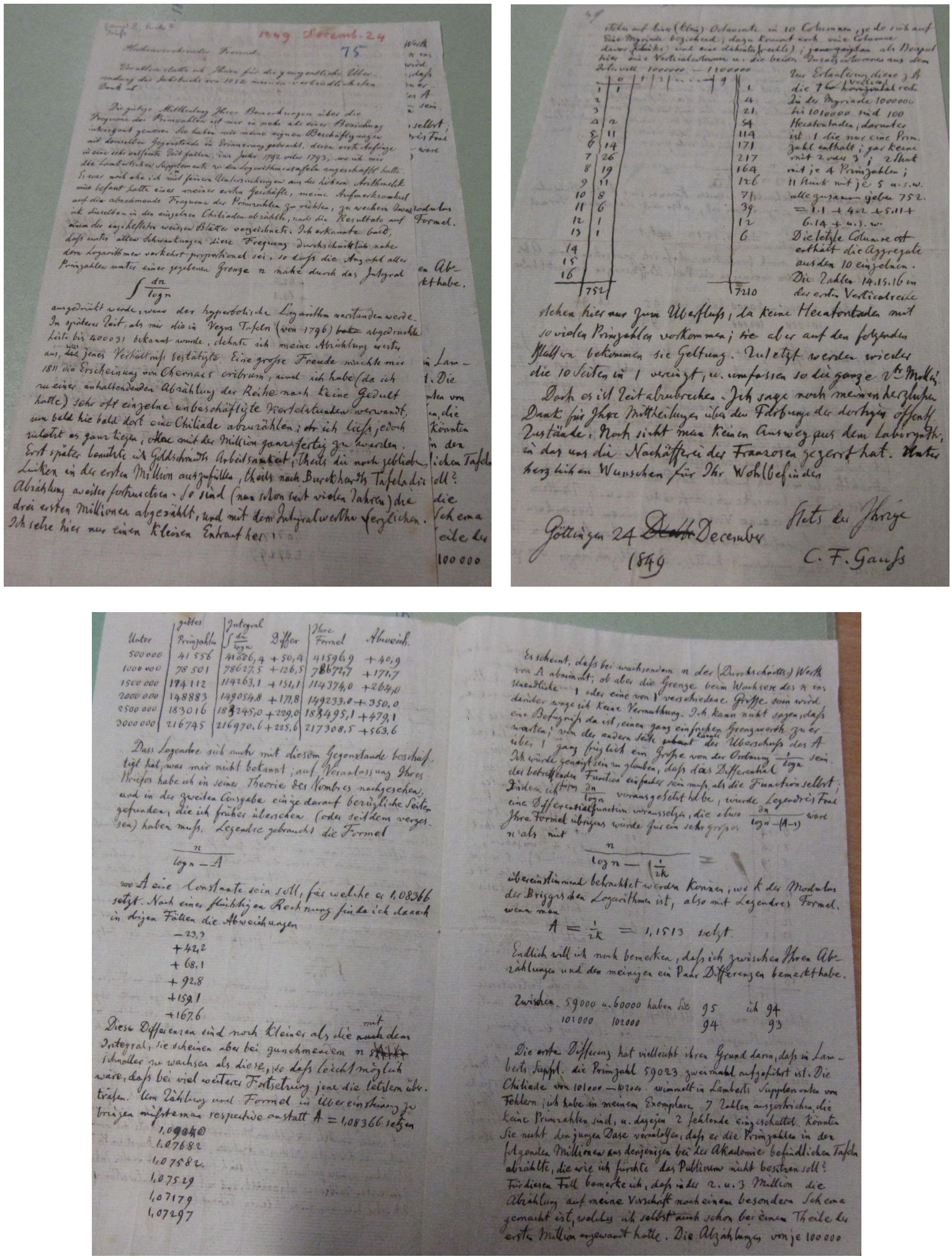

Pages from an 1849 letter written by Carl Friedrich Gauss (1777-1855) to a former pupil regarding the prime number theorem. Manuscript in the Göttingen Library, University of Göttingen. Photographs courtesy of Prof. John Coates. 